\title{
A Comparative Study on the Detection of Intestinal Parasites by Using Different Methods from Stool Sample in a Tertiary Care Centre
}

\author{
Bhawna Kumari, Himadri Dutta* and M. Kalyani
}

Department of Microbiology, Saveetha Medical Collegeand Hospital Saveetha University, Thandalam, Chennai-602105, Tamil Nadu, India

*Corresponding author

K e y w o r d s
$\begin{aligned} & \text { Entamoeba histolytica, } \\ & \text { Giardia lamblia, } \\ & \text { Strongyloides stercoralis, } \\ & \text { Concentration techniques }\end{aligned}$
Article Info
$\begin{aligned} & \text { Accepted: } \\ & 20 \text { March } 2018 \\ & \text { Available Online: } \\ & 10 \text { April } 2018\end{aligned}$

A B S T R A C T
Parasitic infections, caused by intestinal helminths and protozoan parasites, are among the most prevalent infections in developing countries, while in developed countries, gastrointestinal infections are caused more commonly by protozoan parasites compared to helminths. A laboratory analysis of stool samples was done for detection of intestinal parasites in the Microbiology laboratory of a tertiary care hospital for a period of 6 months (February 2017 to July 2017). Stool samples were examined by different methods like direct smears (Saline and Lugol's iodine) and concentration techniques. Formalin - ether sedimentation method and Zinc-sulphate floatation method and modified Ziehl Neelsen staining method. In our study a total of 231 stool samples were analysed and prevalence of intestinal parasitic infection was found to be $13.41 \%$ (31/231). There were five different parasites encountered. The most common parasite identified was Giardia lamblia (52\%), followed by Entamoeba histolytica (19.35\%), Hookworm (16.12\%), Strongyloides stercoralis $(6.45 \%)$ and Cryptosporidium parvum (6.45\%). Use of concentration techniques definitely increased the detection rate when compared to conventional saline and iodine mount examination.

\section{Introduction}

Parasitic infections, caused by intestinal helminths and protozoan parasites, are among the most prevalent infections in developing countries, while in developed countries such infections are caused more commonly by protozoan parasites compared to helminths. Intestinal parasites cause a significant morbidity and mortality in endemic countries. These infections are most prevalent in tropical, sub-tropical regions of the developing world where adequate water and sanitation facilities are lacking (Savioli and Albonico, 2004). Ethnic eating habits, poverty, tourism to exotic areas and environmental degradation and unhygienic living conditions have led to emergence of food- borne parasitic infections (Bundy et al., 1995).

About 3.5 billion people are affected resulting in 450 million cases of illness with the majority being children. These infections lead to iron deficiency anaemia, growth retardation 
in children and other physical and mental health problems and so they represent as serious public health problem. (WHO, 1998) Till now, intestinal parasites such as Ascaris lumbricoides, Entamoeba histolytica, Cyclospora cayetanensis, Giardia lamblia and Hookworms, Crptosporidium spp. are among the major contributors to the global infection burden as they are prevalent in different parts of the world such (Alum et al., 2010). Strongyloidosis a parasitic disease caused by a nematode called Strongyloides stercoralis. It is a disease of worldwide distribution but more common in tropical and subtropical countries (Beaver et al., 1984). Although much less prevalent than either Ascaris or Hookworm, infection with Strongyloides stercoralis totals some 40 million cases in the world (Jones, 1950).

The problem of parasitic infection is more acute in India because of indifferent attitude of the population towards personal hygiene, their low socioeconomic status and low literacy rate.

Major parasitic infection are soil transmitted and food borne helminths like Ascaris, Taenia, and Trichuris trichuria causes wide spread yet often neglected morbidity in infected people particularly in children and pregnant women (Ritchie, 1948).

Amoebiasis occurs also throughout all areas of the world, the causative agent of which is Entamoeba histolytica, the only amoeba pathogenic for humans (Savioli and Albonico, 2004; Bundy et al., 1995; WHO, 1998). In nature, the organism exists in the cyst form. It enters the body through contaminated food or water.

Gastrointestinal infections are very common in patients with HIV infection or AIDS, and diarrhoea is a common clinical presentation of these infections. Acid fast protozoans are very commonly responsible for diarrhoea in HIV positive patients leading death in many cases. Reports indicate that diarrhoea occurs in 30-60 $\%$ of AIDS patients in developed countries, and in about $90 \%$ of AIDS patients in developing countries. (Antony et al., 1988) A number of parasites are now in world-wide in their distribution and many infections once thought to be harmless are now known to be life- threatening in immunocompromised individuals and those suffering from Acquired immunodefiency Syndrome (AIDS).

The usual methods that are used for detection of intestinal parasites from stool specimens are the direct wet mount using saline and iodine, but the detection rate can be enhanced through using concentration procedures such as Salt floatation, Zinc sulphate centrifugal floatation, formal ether concentration (Ritchie, 1948) and modified Baerman sedimentation (Kulkarni et al., 2009).

Microscopic examination is still the gold standard for making a diagnosis for most intestinal parasites to ascertain the presence of parasites in the faeces.

With this background the study was undertaken to detect intestinal parasites by using various methods among the patients attending our Institute.

\section{Materials and Methods}

This study was conducted at Department of Microbiology, Saveetha Medical College and Hospital, Thandalam, Chennai for a period of six months (February 2017 to July 2017). Stool samples were collected in a wide mouthed clean, dry container without preservatives from the 231 patients. Stool samples received in the laboratory were processed immediately thereafter. Stool samples from immunocompromised patients were also included in this study. But the cases 
of bacterial and viral diarrhoea including rice watery stool were excluded from the study. Macroscopic examination was done to look for colour, consistency, presence of mucus and blood, and presence of parasitic structures such as proglottids, scolices, adult tapeworm, enterobius, ascaris, or hookworm. For microscopic examination, Saline wet mount was done to detect protozoal trophozoites and helminthic eggs or larvae and iodine wet mount was done to detect cysts. Stool concentration was done by Formalin-ether sedimentation technique and Zinc sulphate floatation method. (Raza and Sami, 2009) Modified Ziehl Neelsen staining was also done for all samples to detect acid fast parasites (Kumurya and Gwarzo, 2013).

\section{Results and Discussion}

In this study a total number of 231 stool samples were collected from both out patients and in patients of Saveetha Medical College and Hospital, Thandalam a period of 6 months from February 2017 to July 2017.

In this study we have evaluated the individual recovery rate of different stool concentration

Image.1

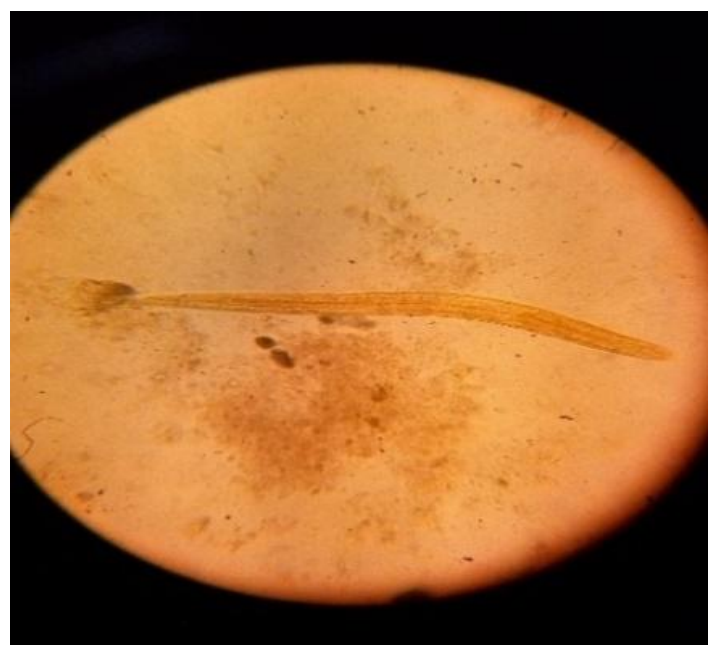

Larva of Strongyloides stercoralis techniques which are used in the detection of intestinal parasites. The techniques evaluated include conventional direct wet mount, formal-ether sedimentation technique, zinc sulphate centrifugal floatation techniques and Modified Ziehl Neelsen's staining method.

Among the 231 samples received in microbiology department, 31(13.4\%) were positive for intestinal parasitic infection. In a similar study conducted by Bineshlal et al., in Vellore (Babiker et al., 2009) the prevalence rate was found to be 34\%.Comparatively lower rate of infection in our study can be explained by gradual improvement of sanitation, drinking water facility and nutritional status among the local population.

In our study, the maximum positive cases were from general medicine department $48.38 \%$ (15/31) followed by paediatric department $38.70 \%$ (12/31). In a similar study rates were recorded by AL-Kubaisy et al., (2014) which showed the largest number of intestinal parasitic infections were found in children aged $<5$ years and that infection declined progressively with increasing age groups.

\section{Image. 2}

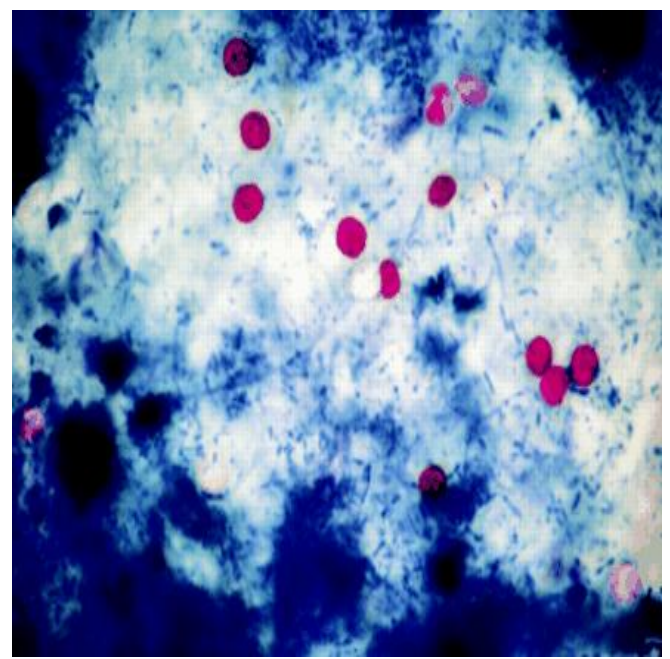

Oocyst of Cryptosporidium parvum 
Table.1

\begin{tabular}{|r|l|c|c|c|c|}
\hline SI. & PARASITES & $\begin{array}{l}\text { TOTAL } \\
\text { SAMIPLES }\end{array}$ & $\begin{array}{l}\text { WET } \\
\text { MOUNT } \\
\text { SMEAR }\end{array}$ & $\begin{array}{l}\text { CONCENTRA TION } \\
\text { MIETHOD }\end{array}$ & $\begin{array}{l}\text { ZIEHL } \\
\text { NEELSEN'S }\end{array}$ \\
\hline 1 & Giardia lamblia & $\mathbf{1 6}$ & $\mathbf{6}$ & $\mathbf{1 0}$ & NA \\
\hline 2 & Entamoebahistolytica & $\mathbf{6}$ & $\mathbf{2}$ & $\mathbf{4}$ & NA \\
\hline 3 & Hookworm & $\mathbf{5}$ & $\mathbf{3}$ & $\mathbf{2}$ & NA \\
\hline 4 & Strongyloides stercoralis & $\mathbf{2}$ & $\mathbf{2}$ & - & - \\
\hline 5 & Cryptosporidium parvum & $\mathbf{2}$ & $\mathbf{2}$ & $\mathbf{N D}$ & $\mathbf{2}$ \\
\hline
\end{tabular}

Our study showed that the prevalence of intestinal parasitic infection was slightly more in females $52 \%(16 / 31)$ than males $48 \%$ (15/31). In a similar studies done in Bihar showed increased infections among females (56\%) (Rituparna et al., 2017). The reason for female being more infected was probably due to the differences in technique used or could be due socioeconomic and nutritional status of the women population which were included in our study.

Among different parasites detected protozoal infection $77.4 \%$ (24/31) was more common compared to helminthic infestation $23 \%$ (7/31). However study done by Kang G et al., whose prevalence rate of protozoal parasites was $(45.38 \%)$ and that of helminthic parasites was (33.84\%) (Kang et al., 1998) As shown in Table 1 Giardia lamblia was found to be the commonest parasites detected with a frequency of 52\% (16/31). Among this, one Giardia lamblia positive case was from HIV patient. This finding was similar to the study of Kang $\mathrm{G}$ et al., where the positivity was (53.8\%) (Kang et al., 1998). Other parasites detected were Entamoeba histolytica 19\% (6/31), Hookworm (16.12\%), Strongyloides stercoralis (6.45\%). Cryptosporidium oocysts (6.45\%) were detected from two cases, one of which was from the immunocompetent healthy child and another was immunocompromised patient with past renal transplant. In a similar study, Kumurya et $a l$., had reported Cryptosporidium oocysts only in $6 \%$ of their HIV positive diarrheal stool samples (Kumurya and Gwarzo, 2013).
In our study the recovery rate for Giardia lamblia by routine direct smear (wet mount and iodine and saline preparations) was only $38 \%$ $(6 / 16)$. But after doing concentration procedures 10 more positive cases were detected which were initially negative by routine wet mount without concentration (Table 1). Similar findings were observed in studies done elsewhere (Raza and Sami, 2009; Tobie et al., 1951). The higher recovery rate than the routine method obtained in our study is because the technique concentrates most ova and cysts (Tobie et al., 1951). Concentration techniques leads to the detection of the organisms which are present in small number, these may be missed by using direct wet mounts. The efficacy of concentration methods in this study is due to ability to detect parasites even if they were in small number, also when larger sample's volume had been examined by concentration methods, they detected more than one parasitic species in the same sample (Raza and Sami, 2009).

Due to centrifugation in a fluid of specific gravity 1.18 , the cysts and ova, being lighter than the suspending fluid float to the top, whereas the heavier debris sinks to the bottom. But the zinc sulphate centrifugal floatation method could not recover the eggs of Strongyloides stercoralis in our study.

Early and prompt diagnosis of parasitic infections is very important as timely treatment of these cases will lead to reduced morbidity and mortality. Further, as demonstrated in our 
study, we can conclude that stool concentration techniques should be adopted routinely, since in case of low parasite load, often the diagnosis is missed by carrying out only by conventional method. Formal-ether sedimentation method is better technique for detection of cysts, eggs and larvae. Recovery of Cryptosporidium parvum in a stool sample from immunocompetent patient is a very striking finding in our study which further re iterates the fact that all stool samples should be screened for these acid fast parasites irrespective of the immune status.

\section{References}

AL-Kubaisy, W., AL-Talib, H., Al-khateeb, A., and Shanshal, MM. 2014. Intestinal Parasitic Diarrhea among Children in Baghdad- Iraq. Tropical Biomedicine. 31(3):499-506.

Alum, A.; Rubino, J.R. and Ijaz, M.K. 2010. The global war against intestinal parasites-should we use a holistic approach? International Journal of Infectious Diseases 14:e732-e738.

Antony M A, Brandt L J, Klein R S, Infectious diarrhea in patients with AIDS Dig, Dis, Sci 1988, 33:1141-1146.

Babiker MA, Ali MSM, Ahmed ES. La Revue de Sante de la Mediterorient 2009; 15: 1095-104.

Beaver PC, Jung RC, Cupp EE. Editors. Clinical Parasitology, Ninth Edition Philadelphia, Lea \& Febiger 1984 P 825.

Bundy D, Hall A, Medley G. Evaluating measures to control intestinal parasitic infection, World Health state, 1995;45.

Jones AC. Clinical studies in human strongly oidiasis Gastroenterology. 16 (4) 1950: 743-56.

Kang G, Mathew MS Rajan DP, Daniel JD, Mathan MM, Mathan vi, et al., The prevalence of intestinal parasites in rural Southern Indians. Trop Med Int Health 1998 3(1): 7075.

Kulkarni SV, Kairon R, Sane SS, Padmawar PS, Kale VS, Thakar MR, et al., Opportunistic parasitic infections in HIV/AIDS patients presenting with diarrhea by the level of immunosuppression. Indian $\mathrm{J}$ Med Res. 2009 Jul; 130:63-6.

Kumurya, AS, and Gwarzo MY. Cryptosporidiosis in HIV infected patients with diarrhea in Kano state, North-Western Nigeria. J AIDS HIV Res. 2013 Aug; 5(8):301-5.

Raza, H.H. and Sami, R. A. 2009. Epidemiological study on gastrointestinal parasites among different sexes, occupations and age groups in Sulaimani district. Journal of Duhok University 12(1):317-323.

Ritchie, L.S. 1948. An ether sedimentation technique for routine stool examination Bull. U.S. Army med. Dep. 8:326.

Rituparna B, Bhattacharya P, Paul UK, Bandyopadhyay A. Prevalence of Intestinal Parasites in a Tertiary Care Hospital in Rural Bihar. Int J Sci Stud 2017; 4(12):89-93.

Savioli, L. and Albonico, M. 2004; Cappello, 2004 Soil-transmitted helminths. Nat Rev Microbiology; 2:619-9.

Tobie J.E., Reardon L.V., Bozicevich J., Chihshih B., Mantel N. A Thomas E.H, 1951. "The efficiency of the zinc sulphate technique in the detection of intestinal protozoa by successive stool examinations", Amer. J. Trop. M.

WHO/World Health Organization 1998. Control of Tropical Diseases. Geneva, Switzerland.

\section{How to cite this article:}

Bhawna Kumari, Himadri Dutta and Kalyani, M. 2018. A Comparative Study on the Detection of Intestinal Parasites by Using Different Methods from Stool Sample in a Tertiary Care Centre. Int.J.Curr.Microbiol.App.Sci. 7(04): 2219-2223. doi: https://doi.org/10.20546/ijcmas.2018.704.252 